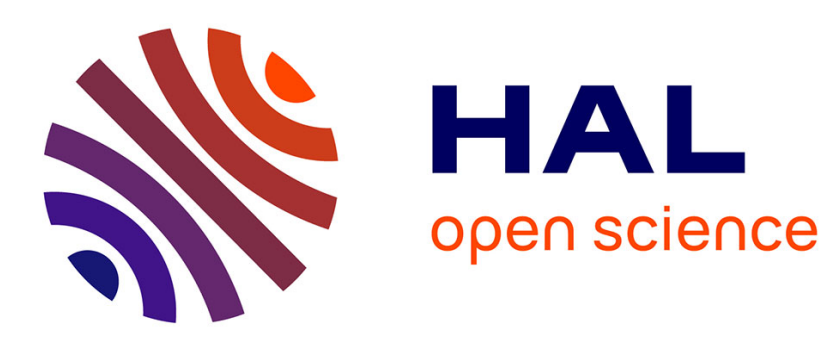

\title{
Investigation of coherency loss by prismatic punching with a nonlinear elastic model
}

Pierre-Antoine Geslin, Benoît Appolaire, Alphonse Finel

\section{To cite this version:}

Pierre-Antoine Geslin, Benoît Appolaire, Alphonse Finel. Investigation of coherency loss by prismatic punching with a nonlinear elastic model. Acta Materialia, 2014, 71, pp.80-88. 10.1016/j.actamat.2014.03.005 . hal-02133735

\section{HAL Id: hal-02133735 \\ https://hal.science/hal-02133735}

Submitted on 27 May 2019

HAL is a multi-disciplinary open access archive for the deposit and dissemination of scientific research documents, whether they are published or not. The documents may come from teaching and research institutions in France or abroad, or from public or private research centers.
L'archive ouverte pluridisciplinaire HAL, est destinée au dépôt et à la diffusion de documents scientifiques de niveau recherche, publiés ou non, émanant des établissements d'enseignement et de recherche français ou étrangers, des laboratoires publics ou privés. 


\title{
Investigation of coherency loss by prismatic punching with a nonlinear elastic model
}

\author{
Pierre-Antoine Geslin*, Benoît Appolaire, Alphonse Finel \\ LEM, Onera/CNRS, 29 avenue de la Division Leclerc, 92322 Chatillon, France \\ Received 12 December 2013; received in revised form 25 February 2014; accepted 2 March 2014
}

\begin{abstract}
Coherency loss of misfitting precipitates by nucleation of dislocations at the interface is investigated using a nonlinear elastic model. This is made possible because the model inherently takes into account, in addition to long-range elastic interactions, phenomena involving dislocation cores such as dislocation nucleation and cross-slip. It is also shown that the model naturally delivers viscous motion laws above a threshold stress. The mechanism of prismatic punching proposed by Ashby and Johnson is confirmed by the present work for small precipitates. For larger precipitates, more complex situations are predicted which involve several dislocations, beyond the scope of the available analyses, but often observed experimentally.
\end{abstract}

(C) 2014 Acta Materialia Inc. Published by Elsevier Ltd. All rights reserved.

Keywords: Nonlinear elasticity; Dislocations; Coherency loss; 3-D Peierls-Nabarro

\section{Introduction}

In the solid state, the coexistence of different phases often generates internal stresses which influence mechanical and physical properties. Hence, understanding how these stresses can be accommodated is an important step in predicting the properties of materials. While, in several systems, accommodation can proceed by solute redistribution in the precipitate vicinity (e.g. [1]), coherency loss processes involving interface dislocations and plastic relaxation of the matrix phase are most frequently encountered.

In diffusion-controlled phase transformation, coherency loss can have a significant influence on the precipitate growth regime. For instance in Ref. [2], the authors show that the lengthening rate of platelet-like $\eta$ and $\theta^{\prime}$ precipi-

\footnotetext{
* Corresponding author.

E-mail addresses: pageslin@gmail.com (P.-A. Geslin), benoit. appolaire@onera.fr (B. Appolaire), alphonse.finel@onera.fr (A. Finel).
}

tates in $\mathrm{Al}-\mathrm{Au}$ and $\mathrm{Al}-\mathrm{Cu}$ alloys is slowed down when precipitates become semicoherent. The presence of interface dislocations reduces the stress field generated by the misfitting precipitate, which provokes a decrease in the driving force, slowing down the precipitate growth. An opposite situation is encountered for the coarsening behavior of $\mathrm{Al}_{3}$ Sc precipitates in Al-Sc alloys [3], where the appearance of interface dislocations increases the precipitate surface energy and increases the coarsening rate.

Generally, the onset of coherency loss is assessed in terms of a simple free energy balance between the contributions from misfit dislocations and the interface energy $[4,5]$. This is due to the complexity and the variety of the mechanisms proposed so far, often based on early post-mortem observations: (i) nucleation and growth of dislocations by the condensation of point defects within the precipitate [6-8]; (ii) attraction of dislocations at interfaces from the bulk matrix [7,9]; and (iii) formation and emission of dislocation loops of prismatic nature (i.e. purely edge loops with their normal parallel to the Burgers vector), also called prismatic punching $[10,8,11]$. As noted in Ref. [12], this last 
process is likely to prevail for small precipitates with strong misfit, in particular when the dislocation density in the matrix is low. Indeed, prismatic punching has been observed in many multiphase materials. This is the case, for example, during precipitation of hydrides in zirconium alloys due to the misfit-generated stresses $[13,14]$, or in metal matrix composites (MMCs) with the build-up of thermal stresses during non-isothermal treatments $[15,16]$. It has been noted in Ref. [17] that a significant hardening can be achieved by such a process in MMCs.

The very first models proposed for coherency loss by prismatic punching are based on analytical expressions for the elastic interactions between a single precipitate and static dislocation loops with simplified geometries $[10,18,19]$. Among them, the model of Ashby and Johnson [10] is the most appealing as testified by its recurrent use in subsequent studies $[12,13,20,21]$. Unfortunately, it relies on a particular sequence of events which is assumed rather than proved: first, a shear dislocation loop nucleates at the interface, expands into the matrix and forms a prismatic loop by cross-slip of its screw segments. Hence, validating this sequence requires a dynamic modeling approach. This would also be beneficial for investigating more complex situations such as rows of loops as observed in, for example, Refs. $[14,15,22]$ or tangles ensuing from the interaction between prismatic loops $[12,14]$.

However, very few attempts to tackle coherency loss have been made with the current modeling techniques. Prismatic punching around an inclusion has recently been obtained with a molecular statics approach in Ref. [23]. However, due to the small length scales available in atomistic simulations, the observed processes are likely to be influenced by the boundary conditions. In Ref. [24], a level-set method accounting for dislocation dynamics has been used to investigate the by-pass mechanisms of dislocations around a misfitting inclusion and the resulting coherency loss of the precipitates. However, this method does not take into account dislocation nucleation and thus cannot be used to investigate prismatic punching mechanisms. Although the phase field modeling in Ref. [25] attempted to account for a change in the nature of the interface with some effective eigenstrain, the actual process was unfortunately not described.

In the present work, we propose a continuum modeling of dislocation dynamics able to describe nucleation, glide and cross-slip of dislocations based on nonlinear elasticity, which is subsequently used to investigate loss of coherency of a misfitting precipitate. This model can be seen as an extension to three dimensions of the Peierls-Nabarro model $[26,27]$.

The paper is organized as follows. We describe the model in Section 2 before presenting its validation in static and dynamic conditions in Section 3. Then, results concerning the loss of coherency by prismatic punching are discussed in Section 4, demonstrating the relevance of our model and bringing new insights into the physical process.

\section{Nonlinear elastic model}

Our model relies on an elastic energy which is periodic with respect to the shear components of the strain tensor. It is comparable to the models of Carpio and Bonilla $[28,29]$ and Onuki and Minami $[30,31]$. Whereas the former authors resort to a discrete atomic lattice, the latter consider a continuous framework, as followed in the present work. Nonetheless, both approaches yield similar equations after discretization. The major advantages of this kind of model over standard phase-field models of dislocations $[32,33]$ are the following: (i) it incorporates naturally dislocation nucleation, a necessary feature for investigating coherency loss mechanisms and (ii) it does not resort to some gradient term which artificially smooths dislocation cores. Finally, it incorporates naturally cross-slip of screw dislocations which is absent in previous phase-field models of dislocations.

Assuming isotropic elasticity, the total elastic energy of the system is written as follows:

$$
\begin{aligned}
\mathcal{F}= & \int d x\left[\frac{\lambda+2 \mu}{2} \sum_{i}\left(\varepsilon_{i i}-\varepsilon_{i i}^{0}\right)^{2}+\frac{\lambda}{2} \sum_{i \neq j}\left(\varepsilon_{i i}-\varepsilon_{i i}^{0}\right)\left(\varepsilon_{j j}-\varepsilon_{j j}^{0}\right)\right. \\
& \left.+\frac{\mu b^{2}}{8 \pi^{2} d^{2}}\left(3-\sum_{i \neq j} \cos \left(\frac{4 \pi}{b / d}\left(\varepsilon_{i j}-\varepsilon_{i j}^{0}\right)\right)\right)\right]
\end{aligned}
$$

where $\lambda$ and $\mu$ are the Lamé coefficients which may be position dependent, $\varepsilon_{i j}$ denote the strain tensor components, $\varepsilon_{i j}^{0}$ are eigenstrain components associated with the microstructure, and $b$ and $d$ are respectively the norm of Burgers vector and the grid spacing. This elastic energy contains the usual harmonic contributions of the diagonal components of the strain tensor, whereas the shear components are embedded into nonlinear periodic potentials accounting for lattice periodicity. For example, if we consider a perfect crystal which is sheared by the quantity $b / 2 d$ on a platelet of height $d$ and normal $n=z$, the expression (1) recovers a minimum energy because of these periodic functions. On its glide plane, a dislocation can then be seen as a frontier between a sheared and an unsheared part of the crystal, both stabilized as minimum energy states (see Fig. 1). The prefactors of the periodic terms are chosen such that, for small deformations, the elastic energy of a linear elastic solid is recovered. Cosines have been chosen for simplicity and generality reasons but they can easily be replaced by more complicated forms such as the $\gamma$-surfaces obtained from

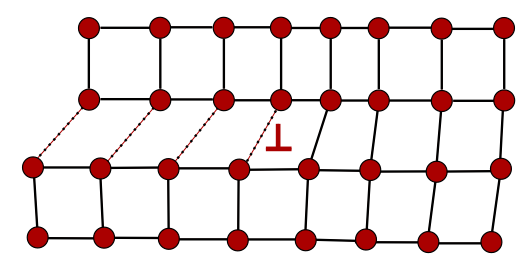

Fig. 1. Edge dislocation seen as a frontier between sheared and unsheared parts of the crystal. 
ab initio calculations. Moreover, the generalization to an elastically anisotropic material is straightforward.

Assuming that small strain theory holds, the strain tensor is defined in a Cartesian framework $\left(x_{i}\right)_{i=1,3}$ as:

$\varepsilon_{i j}=\frac{1}{2}\left(\frac{\partial u_{i}}{\partial x_{j}}+\frac{\partial u_{j}}{\partial x_{i}}\right)$

with respect to the components $u_{i}$ of the displacement field. It is worth mentioning that the choice of small strain is a restriction than can be overcome by following the work of Finel et al. $[34,35]$ on martensitic transformation.

Time-dependent equations for the displacements $u_{i}$ are derived assuming a dissipative dynamics. Indeed, inertial effects can generally be neglected on the dislocation motion when compared to damping caused by the phonon drag mechanisms. For simplicity, external dissipation has been associated with displacements rather than internal dissipation with strains; it is considered that this would not affect the results significantly. Moreover, Langevin noises have been added to introduce random forces in the dynamics that mimick qualitatively thermal fluctuations. Then:

$\eta \frac{\partial u_{i}}{\partial t}=\frac{\partial \sigma_{i j}}{\partial x_{j}}+\xi_{i}$

where $\eta$ is a damping coefficient and $\xi_{i}$ are Gaussian noises satisfying $\xi_{i}(x, t) \xi_{i}\left(x^{\prime}, t^{\prime}\right)=\Gamma \delta\left(x-x^{\prime}\right) \delta\left(t-t^{\prime}\right)$ with $\Gamma$ proportional to the temperature. As usual, the stress field is derived from $\mathcal{F}$ as $\sigma_{i j}=\delta \mathcal{F} / \delta \varepsilon_{i j}$. The kinetic equations for the strain components are easily deduced using Eq. (2):

$\frac{\partial \varepsilon_{i j}}{\partial \tilde{t}}=\frac{1}{2}\left[\frac{\partial \tilde{\sigma}_{i k}}{\partial \tilde{x}_{j} \partial \tilde{x}_{k}}+\frac{\partial \tilde{\sigma}_{j k}}{\partial \tilde{x}_{i} \partial \tilde{x}_{k}}\right]+\frac{1}{2}\left[\frac{\partial \tilde{\xi}_{j}}{\partial \tilde{x}_{i}}+\frac{\partial \tilde{\xi}_{i}}{\partial \tilde{x}_{j}}\right]$

where tildes denote non-dimensionalized quantities $\tilde{x}_{i}=x_{i} / b, \tilde{t}=\eta b^{2} / \mu, \tilde{\sigma}_{i j}=\sigma_{i j} / \mu$ and $\tilde{\xi}=\xi b / \mu$. It is worth mentioning that Eq. (4) guarantee the Saint-Venant compatibility conditions because they derive from the kinetic equations for the displacements.

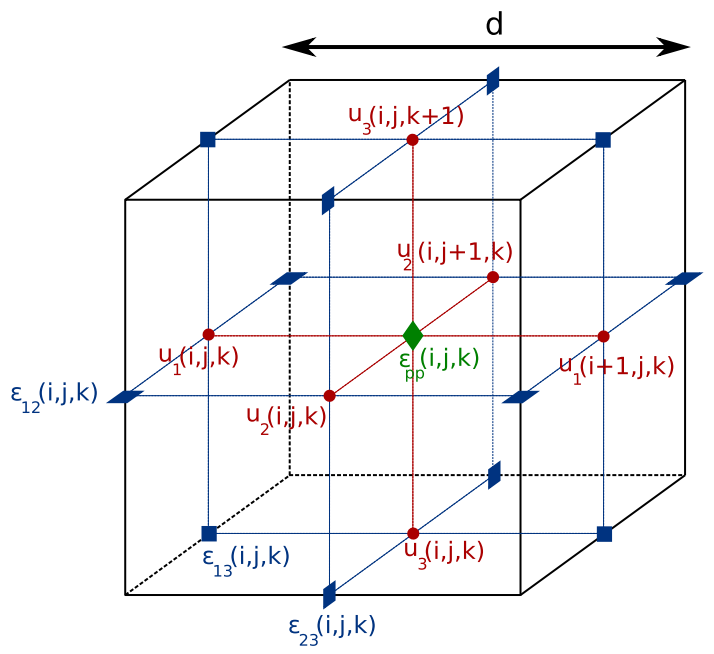

Fig. 2. Staggered finite difference grids.
Eq. (4) are discretized on four staggered grids [36,37] shown with diamonds and squares in Fig. 2: one ${ }^{1}$ for the diagonal components of strain and stress (green diamond), and one for each off-diagonal component (blue squares). The four grids are staggered by a distance $d / 2$ in all three directions where $d$ is the internode spacing in a given grid. The choice of staggered grids has been made to handle sharp variations of coupled strain fields, and prevents the appearance of oscillatory artifacts, as observed, for example, in Ref. [38].

Two kinds of finite differences are defined on the grids. First, forward differences written as $D_{1}^{+} \varphi=[\varphi(i+1, j, k)-$ $\varphi(i, j, k)] / d$, where $\varphi$ is any field, $(i, j, k)$ denotes the coordinates in the grid, and where the subscript of $D$ stands for the direction along which the difference is performed. Second, backward differences are defined by $D_{1}^{-} \varphi=$ $[\varphi(i, j, k)-\varphi(i-1, j, k)] / d$. It is worth mentioning that the use of such operators on staggered grids ensures that only first neighbors are involved in the computational stencils used for differentiation.

First, the components of the stress field are expressed with respect to the components of the strain field on the same respective grids using the homogeneous elastic constants, i.e. $\sigma_{i i}=(\lambda+2 \mu)\left(\varepsilon_{i i}-\varepsilon_{i i}^{0}\right)+\lambda \sum_{j \neq i}\left(\varepsilon_{j j}-\varepsilon_{j j}^{0}\right)$ on the green grid, and $\sigma_{i j}=2 \mu\left(\varepsilon_{i j}-\varepsilon_{i j}^{0}\right)(i \neq j)$ on the three blue grids. Then, the components of the divergence of the stress field are expressed on three auxiliary staggered grids (red circles in Fig. 2) used to define the three components of the displacement field $u$. Storing any symmetric order 2 tensors $\tau$ in vectors as $\left(\tau_{11}, \tau_{22}, \tau_{33}, \tau_{12}, \tau_{13}, \tau_{23}\right)$, the divergence of stress is written in compact form as $\mathbb{D}^{\prime} \cdot \boldsymbol{\sigma}(\boldsymbol{\varepsilon})$ where:

$$
\mathbb{D}^{\prime}=\left[\begin{array}{llllll}
D_{1}^{-} & 0 & 0 & D_{2}^{+} & D_{3}^{+} & 0 \\
0 & D_{2}^{-} & 0 & D_{1}^{+} & 0 & D_{3}^{+} \\
0 & 0 & D_{3}^{-} & 0 & D_{1}^{+} & D_{2}^{+}
\end{array}\right]
$$

The components $\xi_{i}$ of Gaussian noises are also defined on the auxiliary grids associated with the displacement components. Finally, all quantities defined on these auxiliary grids are used for the discretization of the partial differentiation that links the stress components $\varepsilon_{i j}$ to the displacement components $u_{i}$ (Eq. (2)). In compact form, this relation is written as $\boldsymbol{\varepsilon}=\mathbb{D} \cdot u$ with operator $\mathbb{D}$ defined as:

$$
\mathbb{D}=\left[\begin{array}{lll}
D_{1}^{+} & 0 & 0 \\
0 & D_{2}^{+} & 0 \\
0 & 0 & D_{3}^{+} \\
D_{2}^{-} / 2 & D_{1}^{-} / 2 & 0 \\
D_{3}^{-} / 2 & 0 & D_{1}^{-} / 2 \\
0 & D_{3}^{-} / 2 & D_{2}^{-} / 2
\end{array}\right]
$$

Hence, after discretization Eq. (4) reads:

$\dot{\epsilon}=\mathbb{D} \cdot \mathbb{D}^{\prime} \cdot \boldsymbol{\sigma}(\boldsymbol{\varepsilon})+\mathbb{D} \cdot \xi$

\footnotetext{
${ }^{1}$ For interpretation of color in Figs. 2, 5 and 9, the reader is referred to the web version of this article.
} 
Time integration of the resulting system of ordinary differential equations is performed with a simple explicit Euler scheme:

$\boldsymbol{\varepsilon}^{t+d t}=\boldsymbol{\varepsilon}^{t}+d t\left(\mathbb{D} \cdot \mathbb{D}^{\prime} \cdot \boldsymbol{\sigma}\left(\boldsymbol{\varepsilon}^{t}\right)+\mathbb{D} \cdot \xi^{t}\right)$

where the superscript indicates the time step at which the quantity is assessed.

Periodic boundary conditions have been considered for simplicity. Moreover, the model has been implemented in a finite-difference code parallelized using the MPI standard.

It must be stressed that the choice of components of the strain tensor entering the periodic potential in Eq. (1) must comply with the grid. Indeed, whereas the grid imposes the glide planes, the arguments of the periodic potentials are linked to the glide directions, i.e. possible Burgers vectors in these planes. In the present case, the glide planes are those of a simple cubic structure, i.e. $\{100\}$, and the cosines render $\langle 100\rangle$ the easiest directions for gliding. More common crystal structures, i.e. body-centere cubic (bcc), facecentered cubic (fcc) and hexagonal close-packed (hcp), would necessitate grids that display the symmetries of the glide systems, and a natural choice would be to conform to the atomic lattice as in Ref. [29].

\section{Validation}

First, the stress field around an edge dislocation is compared to the classical solution of linear elasticity, for two grid spacings $d=b$ and $d=10 b$. For that purpose, a static dislocation dipole is introduced in a large simulation cell and the elastic energy (Eq. 1) is relaxed using the dissipative dynamics (Eq. 4). The stress components $\sigma_{12}$ and $\sigma_{11}$ are plotted in Fig. 3a and b. Their profiles across the dislocation core along the $x$ and $y$ axes respectively, compare very well to the analytical solution (black dashed line) for the far fields decaying as $1 / r$. Close to the dislocation, the nonlinear part of the elastic energy introduces a dislocation core and avoids the singularity of the analytical solution. For $d=b$, and $v=1 / 3$, the dislocation core width is $w=1.3 b$, close to the value found by Peierls [26], i.e. $w_{p}=1.5 b$. It must be noted that this value is small when compared to dislocation cores in fcc or bcc lattices where dislocations are generally dissociated into partials. However, recovering such a dissociation is not an issue with the present model provided that a more general periodic function is employed based on generalized stacking fault energy. For $d=10 b$, the core is much wider and spans over $13 b$. Correlatively, the maximum stress at the dislocation is 10 times smaller than for the fine grid. As expected, discretization has a great impact on the core structure. Similar statements can be made for screw dislocations.

Second, the model is examined in dynamic situations. The velocity of a single dislocation, either edge or screw, is determined for different applied shear stresses. The resulting curves are shown in Fig. 4 for two grid spacings, $d=b$ and $d=10 b$. In all cases, a threshold stress (Peierls stress) must be overcome to move the dislocations; indeed, due to the discretization, the dislocations experience a pinning effect below the threshold. In the work of Peierls [26] and Nabarro [27] (PN model), the Peierls stress is found by introducing discreteness on the position of the atomic halfplanes located above and below the glide plane. The energy of the dislocation function of its position (i.e. the Peierls energy) is calculated by summing the energy on both upper and lower atomic half-planes and displays a period $b / 2$. The fact that the dislocation recovers a minimum of energy after being displaced by $b / 2$ has been noted to be unrealistic [39]. Other derivations [39-41] of the Peierls stress consist in performing the summation of the Peierls energy on the atomic bounds linking the atoms of both sides of the glide plane. This approach leads to the expected $b$-periodicity for the Peierls energy. In our case, the numerical values of the Peierls stress should be compared to this later derivation since the lattice friction experienced by the dislocation comes from the discretization of the stress fields that can be seen as interatomic bounds.
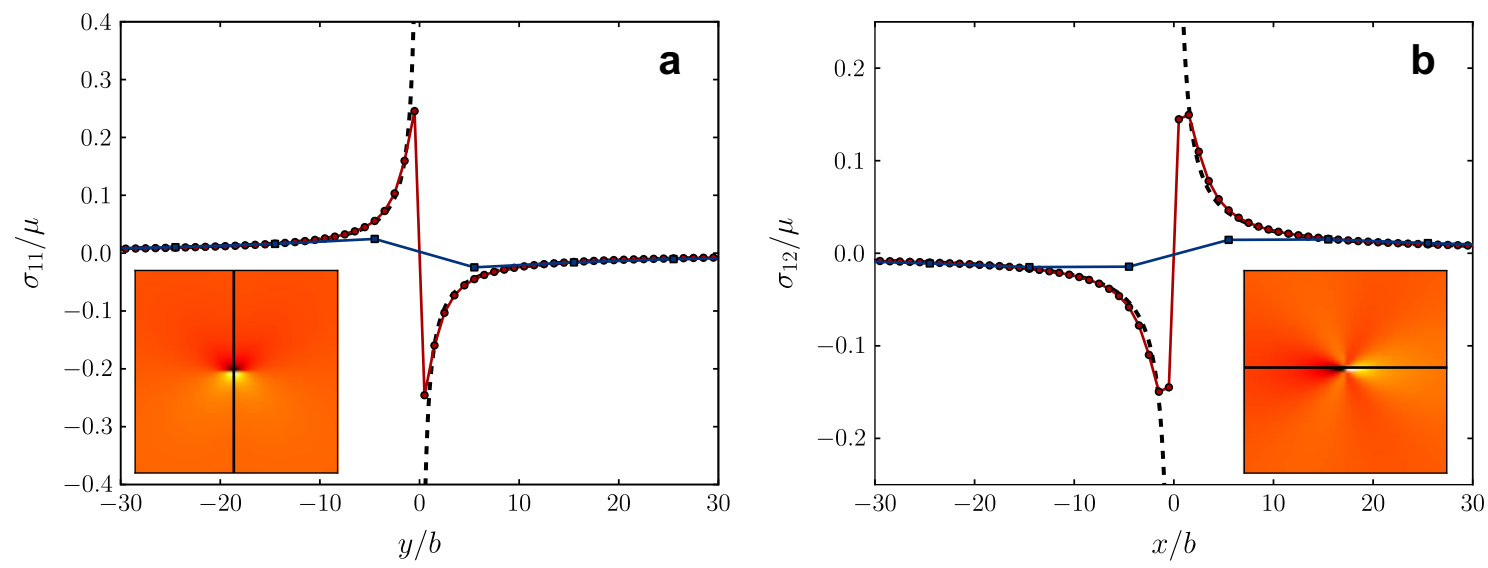

Fig. 3. components (a) $\sigma_{12}$ and (b) $\sigma_{11}$ of the stress tensor around an edge dislocation with $d=b$ (red circles) and $d=10 b$ (blue squares). The black dashed line shows the analytical solution of linear elasticity. (For interpretation of the references to colour in this figure legend, the reader is referred to the web version of this article.) 


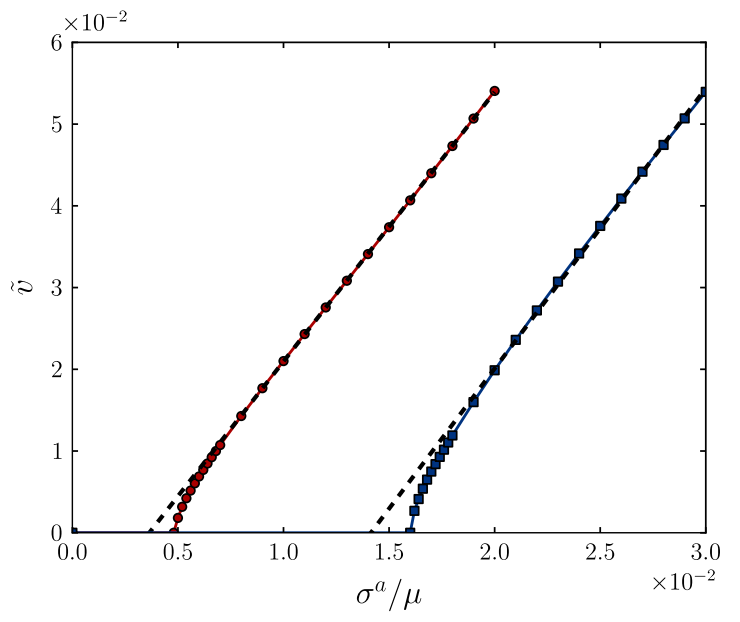

Fig. 4. Normalized velocity vs. normalized applied stress of edge (red circles) and screw (blue squares) dislocations for $d=b$. (For interpretation of the references to colour in this figure legend, the reader is referred to the web version of this article.)

The values of the Peierls stress found with our numerical model are reported in Table 1 and compared to the analytical expression taken from Ref. [41]. The differences between the values corresponding to $d=b$ and the PN model can be attributed to some limitations of the PN model that are lifted in our model: (i) the shear elastic energy appears twice in the PN model, once in the elastic bulk contribution and once in the periodic potential; (ii) in the PN model, the periodic potential appears solely in the glide plane, while it is active on every other parallel plane in our model - consequently, the dislocation core can also spread in the direction normal to the glide plane; (iii) in the PN model, the elastic displacements are assumed to be stress independent when computing the Peierls stress, while our model takes into account the relaxation of these displacements. For $d=10 b$, the Peierls stresses are divided by 10 , as a direct consequence of the drop of the maximum stress at the dislocation. Above the Peierls stress, linear relationships between the dislocation velocities and the applied stress are rapidly observed after small nonlinear regimes. These linear regimes correspond to the usual law of motion $v=b \sigma / B$, where $B$ is the phonon drag coefficient. It is worth noting that the slopes, i.e. $b / B$, are the same for a given viscosity $\eta$ irrespective of the nature of the dislocation and the grid spacing. Moreover, a linear relationship exists between $\eta$ and $B$. Thus, one can choose

Table 1

Normalized Peierls stress $(v=1 / 3)$.

\begin{tabular}{|c|c|c|}
\hline & Edge & Screw \\
\hline $\begin{array}{l}\text { Present model } \\
d=b \\
d=10 b\end{array}$ & $\begin{array}{l}4.72 \times 10^{-3} \\
4.72 \times 10^{-4}\end{array}$ & $\begin{array}{l}1.59 \times 10^{-2} \\
1.59 \times 10^{-3}\end{array}$ \\
\hline $\begin{array}{l}\text { Peierls-Nabarro } \\
1 / a \exp (-\pi / a) \\
\text { with } a\end{array}$ & $\begin{array}{l}1.35 \times 10^{-2} \\
1-v\end{array}$ & $\begin{array}{l}4.32 \times 10^{-2} \\
1\end{array}$ \\
\hline
\end{tabular}

the numerical value of the damping coefficient $\eta$ in order to reproduce a physical drag coefficient.

In the following, a grid spacing $d=10 b$ is used to handle large precipitates with radii up to $400 b$, which are generally beyond the reach of atomic-scale methods (e.g. [23]). Moreover, with this discretization, the Peierls stresses are of the order of $10^{-3} \mu$ closer to realistic values than with $d=b$. Finally, for the coarser grid, the stress necessary to nucleate a dislocation loop is $\mu b /(2 \pi d) \simeq 1.6 \times 10^{-2} \mu$, a realistic value when nucleation is assisted by thermal fluctuations [42].

\section{Results}

We first investigate the process of coherency loss by prismatic punching. A schematic mechanism has been proposed by Ashby and Johnson [10] and is reproduced in Fig. 5a. We consider a misfitting spherical precipitate of radius $R$ (red). According to Eshelby's solution [43], the shear stress is maximum on planes defined by $x, y, z= \pm R / \sqrt{2}$ and forming the parallelepiped (often called the glide cylinder) shown in Fig. 5a. Consequently, a dislocation shear loop (blue) is more likely to nucleate on one of these planes (e.g. the top plane). This loop bulges out until it reaches a critical size with screw segments parallel to the edges of the parallelepiped. Then, the screw segments cross-slip down the vertical sides of the parallelepiped until they merge at the bottom of the precipitate and form a prismatic dislocation loop. This prismatic loop is punched out due to the stress field generated by the precipitate. We note that the complementary loop remaining at the precipitate interface is not shown as in Ref. [10].

In order to see whether this process can be reproduced with our model, a precipitate of radius $R=200 b$ with a dilatation eigentrain $\varepsilon_{i i}^{0}=1.75 \%$ is considered (orange in Fig. $5 b$ ) in a large $1280 \times 1280 \times 1280 b^{3}$ system. The precipitate shape is defined with a static phase field: the matrix and precipitate phases are defined a priori and no thermodynamics is associated with their evolution. However, let us highlight that it would be straightforward to embed the phase field into a free energy functional and make it evolve according to an adapted time-dependent Ginzburg equation, but this is not the purpose of this paper. To avoid the simultaneous nucleation of shear loops on symmetric locations, the misfit is chosen just below the critical misfit for spontaneous nucleation. Moreover, to prevent nucleation on asperities at the interface induced by the grid, the precipitate/matrix interface is smoothed over four grid spacings (i.e. 40b). Both the matrix and the precipitate have been attributed the same isotropic elastic constants with $\lambda=2 \mu$. Four snapshots of the process predicted by our model are shown in Fig. 5b. The level-set corresponding to $12 \%$ of the maximum energy is shown in blue to locate the dislocation loop.

Thanks to the Langevin noise $\xi_{i}$ in Eq. (3), a single shear loop with a Burgers vector along the [001] direction nucleates. Once the loop is nucleated, the noises are shut down 

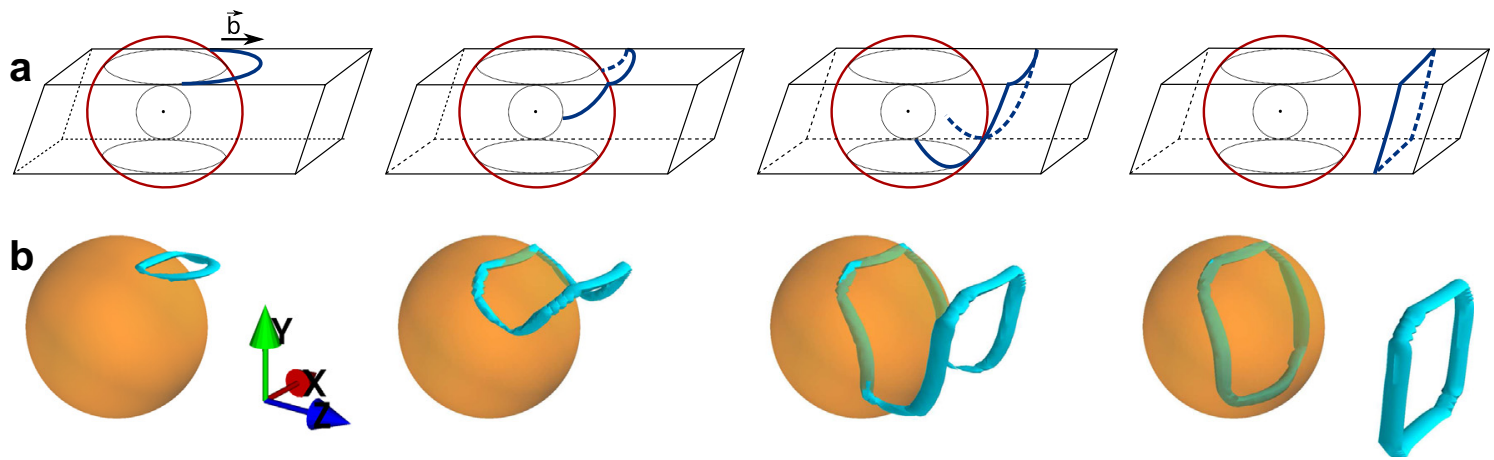

Fig. 5. Prismatic punching mechanism: (a) as described in Ashby and Johnson [10]; (b) resulting from the model $(\tilde{t}=20,80,140$ and 800 after nucleation).

so as not to influence the growth process. The subsequent stages are in good agreement with the picture proposed by Ashby and Johnson. First, the shear loop expands and reaches a critical size at which cross-slip of the screw segments can proceed. Finally, a complete interstitial loop is formed and is punched out from the precipitate. The loop remaining at the surface of the precipitate penetrates a few grid spacings $(\sim 20 b)$ into the diffuse interface to better relax the stress field. It must be stressed that screw dislocations can cross-slip easily in the present model: the mechanism is simply controlled by the local shear stress and is triggered when it reaches a threshold value, without any modification of the configuration of the dislocation core. This is different from the real processes in fcc crystals where the dislocations are dissociated. Indeed, partials have to be constricted to be able to cross-slip and to change glide plane [39]. Thus, the mechanism proposed by Ashby and Johnson is likely to be operative in systems displaying only simple cross-slip processes. Nonetheless, accounting for partials and complex cross-slip could be achieved in the present modeling framework by replacing the simple cosine potential by complex $\gamma$-surface energies and adopting discretization grids displaying the fcc symmetry.

Our process exhibits a few differences with the process proposed by Ashby and Johnson, as shown in Fig. 6 with the front view (i.e. projection on $(x, z)$ ) of the punchedout loop. (i) Because of the diffuse interface, the region of maximum shear stress where the first loop has nucleated is not located exactly at $z=R / \sqrt{2}$ as predicted by Eshelby's solution but ahead of the interface at $z=0.825 R$ ( 2 grid spacings above). (ii) The prismatic loop exhibits blunted corners unlike the parallelepipedic loop in the scheme of Ashby and Johnson (Fig. 5). This difference can be attributed to the difference in the respective stress fields: whereas only the stress field generated by the misfitting precipitate is considered in Ref. [10], our calculations take into account all the elastic interactions, i.e. between the dislocation and the precipitate as well as between the different segments of the dislocation. Hence, when the shear loop reaches its critical size, the screw segments experiencing the complex and evolving stress field move by successive elementary (i.e. one grid spacing $d$ ) cross-slip events in alternating perpendicular directions. The resulting step-like

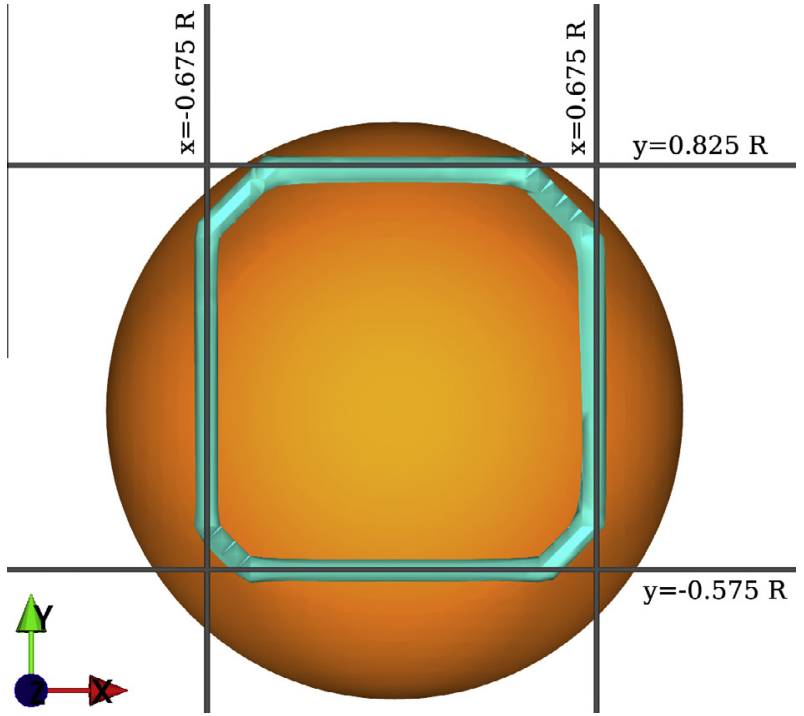

Fig. 6. Front view of the last snapshot in Fig. 5 (time $\tilde{t}=800$ after nucleation). Black lines indicate the principal glide planes of the prismatic loop (glide cylinder).

cross-slip, smoothed by the interpolations of the visualization software in Fig. 6, explains the shape of the loop corners. (iii) As observed in Fig. 5, the prismatic loop is not centered with respect to the spherical precipitate. This results again from the perturbation of the stress field by the dislocation which shifts the maximum shear stress in planes at $z \approx-0.5 R>-0.825 R$.

Beyond the previous mechanism, more complex situations can be encountered which cannot be rationalized with tractable analytical solutions. First, large precipitates can induce multiple prismatic punching as shown in Fig. 7. The calculation has been carried out considering a precipitate with $R=320 b$ and $\varepsilon_{i i}^{0}=1.55 \%$. As in the Ashby-Johnson mechanism, the loss of coherency starts with the nucleation of a shear loop and its subsequent expansion. Because the loop is large, the cross-slip of the screw segments gives rise to a complex process: the screw segments roll up (Fig. 7b) to give small shear loops (Fig. 7c). These secondary loops expand due to the stress generated by the misfitting precipitate and merge to give a second 

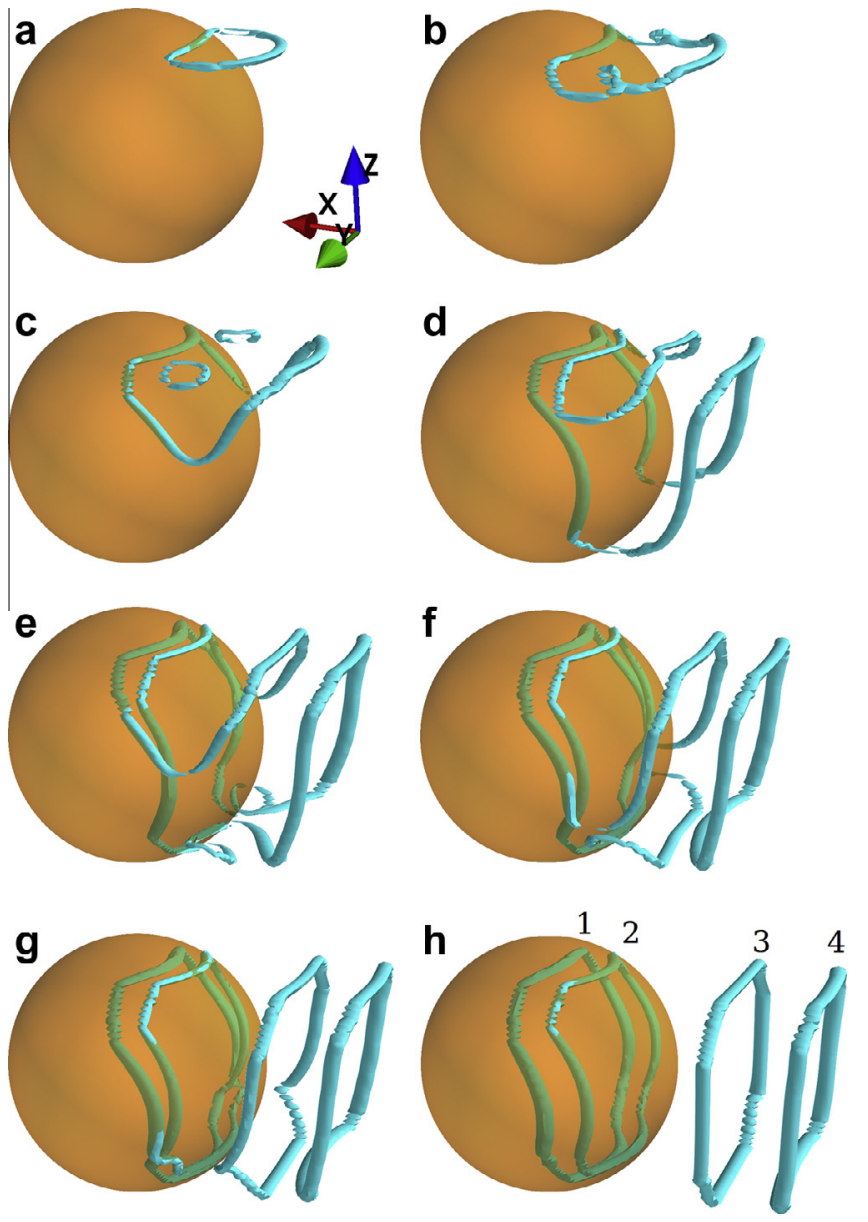

Fig. 7. Double prismatic punching from a precipitate with radius $R=320 b$ and with $\varepsilon_{i i}^{0}=1.55 \%$. Snapshots at times $\tilde{t}=16,40,64,104$, 136, 160, 176, 200 after nucleation.

"inner" shear loop (Fig. 7d). This "inner" loop reacts with the primary loop that lies on the precipitate surface and recombines with it (Fig. 7e). When the screw segments of the inner shear loop start to cross-slip, the screw segments of the first loop have already reached the lower part of the glide cylinder and start to join in a more complex way than during the Ashby-Johnson mechanism (Fig. 7e). Indeed, after their cross-slip, the screw segments of the "outer" loop exhibit a roll-up mechanism similar to the one described above and which, by splitting, leads to the formation of two small shear loops. Afterwards, these small loops merge together and with the bottom part of the first complementary loop (Fig. 7e and f). This leads to the formation of a protruding dislocation component on the bottom part of the first loop that finally merges with the inner loop (Fig. 7f and g). This last mechanism leads to the formation of the second punched-out prismatic loop (Fig. 7h). It can be noticed that the glide cylinders of the loops are not identical: complementary loops 1 and 3 are larger than complementary loops 2 and 4 in Fig. 7 h.

A second situation where loss of coherency is more complex than the Ashby-Johnson scenario arises when the misfit is large enough to promote the nucleation of many dislocations at the precipitate interface. In that case, the formation of short helices, i.e. two prismatic loops linked together, is often observed as shown in Fig. 8a for a precipitate radius $R=360 \mathrm{~b}$ and $\varepsilon_{i i}^{0}=2.5 \%$ (to make the picture clearer only one helix is shown). This configuration is very close to what is observed experimentally around hydrides in $\mathrm{Nb}$, as indicated by arrows in Fig. 8b [14]. This mechanism differs from the processes leading to long helical dislocations that result from the absorption of vacancies by a screw dislocation or the interaction between a sequence of prismatic loops with a screw dislocation line [44,45]. The present calculations clearly show that in our case the short helices are due to the asymmetry and complexity in the nucleation process occurring at the interface and are not occurring following the creation of the loops.

Most often, prismatic punching generates trains of loops aligned along the glide cylinders, as shown in Fig. 8b. It can be shown that the number $N$ of loops depends on the size and on the eigenstrain of the precipitate. Indeed, $N$ can
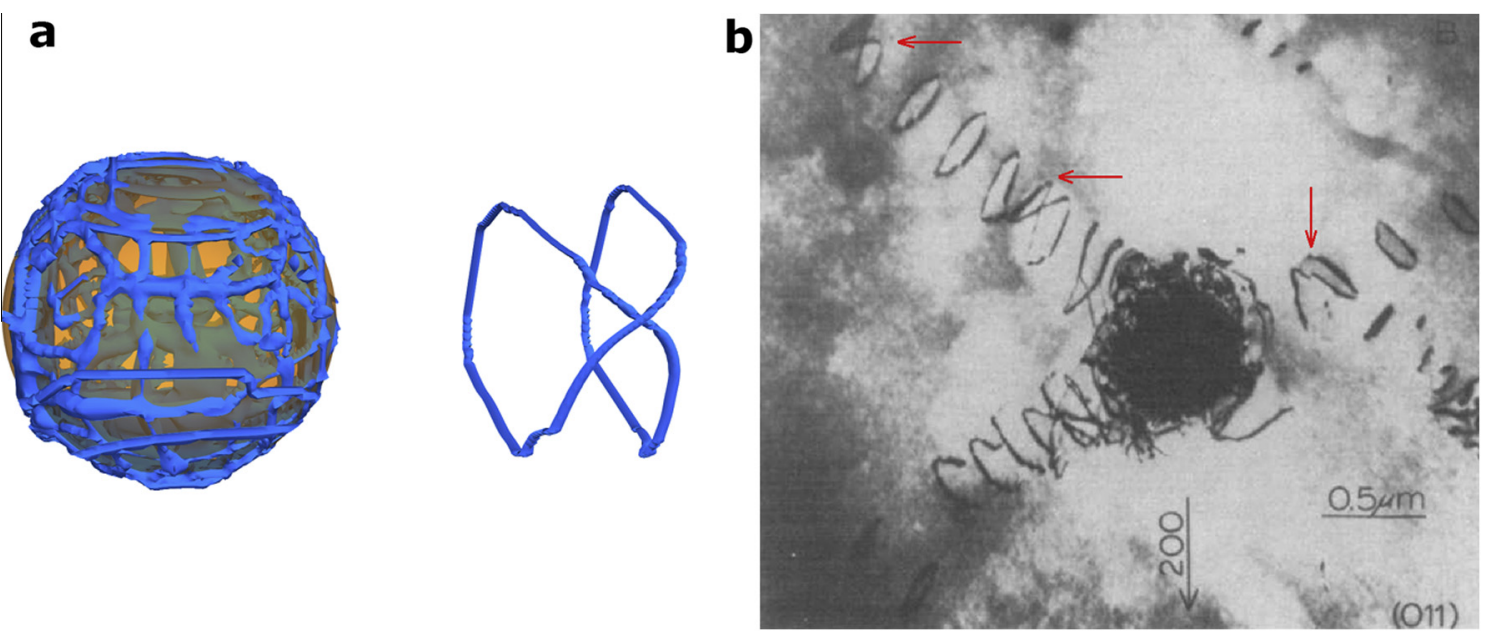

Fig. 8. (a) Two prismatic loops linked together forming a short helix $\left(R=360 \mathrm{~b}\right.$ and $\left.\varepsilon_{i i}^{0}=2.5 \%\right)$. (b) TEM micrograph of an hydride precipitate in Nb (from Ref. [14]) where helices are indicated by arrows. 


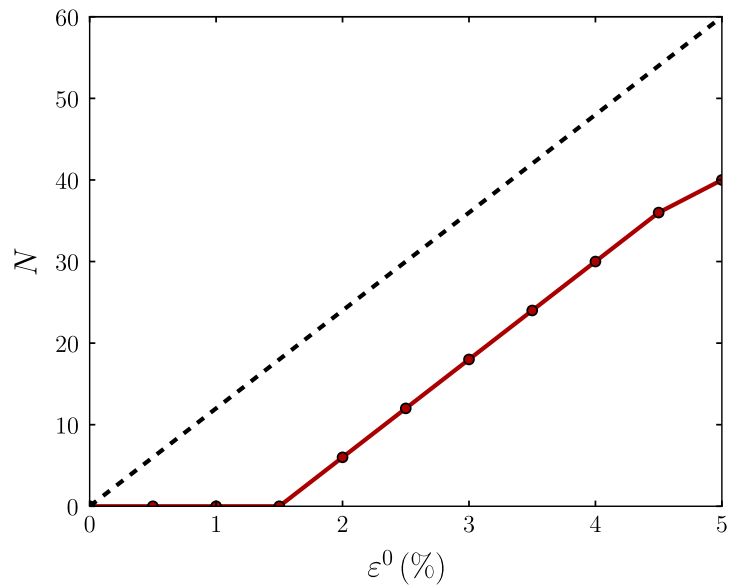

Fig. 9. Number of punched-out dislocations vs. eigentrain for $R=200 b$ (red dotted line). The estimate of Eq. (9) is shown by the black dashed line.

be crudely estimated by assuming that the dislocations left on the precipitate relax entirely the misfits in the directions of emission [16]. For a spherical precipitate of radius $R$ with a dilatation eigenstrain $\varepsilon^{0}$ and emissions along three orthogonal directions:

$N=3 \times \frac{2 R \varepsilon^{0}}{b}$

Although this rough estimate does not take into account the elastic interactions and neglects the threshold stress for nucleation, it is often used to assess the size of the plastic zone around misfitting inclusions and precipitates (e.g. $[46,47])$. To check the trend predicted by Eq. (9), i.e. the linear increase of $N$ with $\varepsilon^{0}$ (the linear increase with $R$ is more difficult to investigate due to limitations in computational resources), we have carried out a series of calculations considering a precipitate with $R=200 \mathrm{~b}$ and with various eigenstrains. A large simulation box $2560 \times 2560 \times 2560 b^{3}$ has been chosen such that the interactions with periodic images are sufficiently small.

The number of loops emitted from the precipitate is plotted as a function of $\varepsilon^{0}$ in Fig. 9 (red dotted line). $N$ increases linearly with the eigenstrain with a slope in agreement with the simplified estimate. The difference between both lines is due to nucleation: below a threshold eigenstrain of $1.5 \%$, no dislocation can nucleate for $R=200 \mathrm{~b}$. Moreover, above 35 loops, stresses from periodic images become important and inhibit the nucleation of further dislocations: for large $\varepsilon^{0}$ (around 5\%), $N$ deviates from its linear dependence with the eigenstrain.

\section{Discussion}

The above results demonstrate that our model has great potential for investigating many phenomena involving phase transformations and dislocations. However, its current implementation, which relies on a cubic grid and a cosine for the periodic function, restricts its application to the glide systems of a simple cubic lattice, and consequently its generalization to fcc, bcc or hcp lattices is necessary to capture more realistic phenomena. As briefly mentioned above, this generalization requires: (i) identifying the relevant strain components entering the periodic potentials which must comply with the glide directions and (ii) discretizing the partial differential Eqs. 4 on a grid displaying the same planes as dislocation glide. The first item is straightforward as it can easily be achieved by expanding the relevant $\gamma$-surface into a Fourier series. The second item is slightly involved in terms of numerical implementation because the grid structure has to conform to the atomic lattice, at least for fcc, hcp and bcc. For example, for fcc and hcp, triangular (2-D) or tetrahedral (3-D) finite-difference grids must be used.

In this work, we have studied the influence of frozen microstructures on the nucleation of dislocations and their subsequent glide and cross-slip. However, dislocations are also known to promote or inhibit interface movements, either when the transformations are diffusion controlled $[3, \underline{48}-50]$, or when they are displacive [51,52]. Indeed, as shown above in a particular example, the stress fields generated by interface dislocations are significant and may have a considerable impact on the transformation kinetics as well as on the precipitate morphologies. Hence, the straightforward coupling of the present model with a phase-field approach for handling interface movements would bring new insights into the mechanisms of phase transformations at the solid state in crystalline materials.

In particular, it has been suggested $[21,53]$ that interface dislocations contribute significantly to the precipitation of $\gamma$-hydride. It is assumed that the phase change from the hcp $\zeta$-hydride to fcc $\gamma$-hydride is performed through shearing of the precipitate by partial Shockley dislocations. Alongside this process, the hydrogen composition of the precipitate increases and the precipitate grows [53]. This complex phase change involving a diffusive phase transformation as well as dislocation nucleation and motion is beyond the reach of the standard phase-field method $[54,55]$ but could be investigated with the present model coupled with a phase-field approach.

At the time and temperature scales involved in diffusive phase transformations, dislocation climb by vacancy diffusion and absorption is activated and likely to play a significant role. In a recent contribution [56], we propose a phase-field model for dislocation climb based on vacancy absorption and emission. This model can be coupled to a phase-field model for dislocation glide and then provide a complete and coherent framework to treat interactions between dislocation motion and precipitate evolution.

Finally, it can be stressed that besides improving the current model, it is straightforward to use it for investigating void growth by prismatic punching, without resorting to analytical solutions available only for prescribed and oversimplified shapes as in Ref. [57], and at length scales much larger than what can be achieved with atomic-scale models as done in Ref. [58]. 


\section{Conclusion}

We have developed a continuum model for dislocations that inherently accounts for their nucleation, cross-slip and interactions without introducing any ad hoc rules. Careful examinations of its prediction in static and simple dynamic situations have shown that it delivers the expected results: (i) the nonlinearity of the elastic energy naturally gives rise to a dislocation core and prevents the divergence encountered in the classical models for dislocations that rely on linear elasticity; (ii) long-range elasticity is recovered a few grid spacings from the dislocation core; and (iii) linear motion laws are obtained above a threshold stress commensurate with the Peierls stress, in accordance with the viscous dynamics of dislocations.

We have used this model to investigate coherency loss mechanisms of misfitting precipitates by punching of prismatic dislocation loops. We have confirmed that, in the simplest situation where only one prismatic loop is emitted, the coherency loss mechanism follows qualitatively the process proposed by Ashby and Johnson [10]. Quantitative differences have been observed with the original scheme which can be attributed to the complex interactions between prismatic loops and precipitate. Beyond the elementary process, we have been able to study more complex situations involving several loops. In particular, it has been shown that large loops emitted by large precipitates can evolve into two prismatic loops via a complex process involving recombinations of different segments. When many loops nucleate at the interface, short helical loops appear in agreement with experimental observations. Finally, we have shown that trains of loops are well reproduced and follow the expected trends.

\section{Acknowledgments}

Fruitful discussions with Yann Le Bouar are gratefully acknowledged.

\section{References}

[1] Steinbach I, Apel M. Acta Mater 2007;55:4817-22.

[2] Sankaran R, Laird C. Acta Metall 1974;22:957-69.

[3] Iwamura S, Miura Y. Acta Mater 2004;52(3):591-600.

[4] Jesser W. Philos Mag 1969;19(161):993-9.

[5] Romanov A, Wagner T, Rühle M. Scripta Mater 1998;38(6):869-75.

[6] Baker R, Brandon D, Nutting J. Philos Mag 1959;4:1339.

[7] Weatherly G, Nicholson R. Philos Mag 1968;17(148):801-31.

[8] Brown L, Woolhouse G. Philos Mag 1970;21(170):329-45.

[9] Link T, Epishin A, Fedelich B. Philos Mag 2009;89:1141-59.

[10] Ashby M, Johnson L. Philos Mag 1969;20(167):1009-22.

[11] Weatherly G. Philos Mag 1968;17(148):791-9.
[12] Johnson W, Lee J. Acta Metall 1983;31(7):1033-45.

[13] Carpenter G, Watters J, Gilbert R. J Nucl Mater 1973;48:267-76.

[14] Makenas B, Birnbaum H. Acta Metall 1980;28(7):979-88.

[15] Dunand D, Mortensen A. Acta Metall Mater 1991;39(7):1417-29.

[16] Dunand D, Mortensen A. Acta Metall Mater 1991;39(2):127-39.

[17] Arsenault R, Shi N. Mater Sci Eng 1986;81:175-87.

[18] Bullough R, Newman R. Philos Mag 1960;5(57):921-6.

[19] Brown L, Woolhouse G, Valdrè U. Philos Mag 1968;17(148):781-9.

[20] Carpenter G. J Nucl Mater 1978;73:190--197.

[21] Carpenter G. Acta Metall 1978;26(8):1225-35.

[22] Tan T, Tice W. Philos Mag 1976;34(4):37-41.

[23] Tsuru T, Shibutani Y. J Phys D Appl Phys 2007;40:2183-8.

[24] Quek S, Xiang Y, Srolovitz D. Acta Mater 2011;59(14):5398-410.

[25] Appolaire B, Aeby-Gautier E, Da Costa Teixeira J, Dehmas M, Denis S. Philos Mag 2010;90(1-4):461-83.

[26] Peierls R. Proc Phys Soc 1940;52(1):34-7.

[27] Nabarro F. Proc Phys Soc 1947;59:256.

[28] Carpio A, Bonilla L. Phys Rev Lett 2003;90(13):135502.

[29] Carpio A, Bonilla L. Phys Rev B 2005;71(13):134105.

[30] Onuki A. Phys Rev E 2003;68(6):061502.

[31] Minami A, Onuki A. Phys Rev B 2005;72(10):100101.

[32] Wang Y, Jin Y, Cuitino A. Acta Mater 2001;49:1847-57.

[33] Rodney D, Le Bouar Y, Finel A. Acta Mater 2003;51:17-30.

[34] Finel A, Le Bouar Y, Gaubert A, Salman O. C R Phys 2010;11(3-4): 245-56.

[35] Salman O. Modeling of spatio-temporal dynamics and patterning mechanisms of martensites by phase-field and Lagrangian methods. Ph.D. thesis, Université Pierre et Marie Curie; 2009.

[36] Saenger E, Gold N, Shapiro S. Wave Motion 2000;31(1):77-92.

[37] Carcione J, Herman G, Ten Kroode A. Geophysics 2002;67(4):1304-25.

[38] Li Y, Hu S, Henager C, Deng H, Gao F, Sun X, et al. J Nucl Mat 2012;427(1-3):259-67.

[39] Hirth J, Lothe J. Theory of dislocations. New York: McGraw-Hill; 1968.

[40] Christian J, Vitek V. Rep Prog Phys 1970;33(1):307.

[41] Joós B, Duesbery M. Phys Rev Lett 1997;78(2):266-9.

[42] Aubry S, Kang K, Ryu S, Cai W. Scripta Mater 2011;64(11):1043-6.

[43] Eshelby J. Proc Phys Soc Lond 1957;241(1226):376-96.

[44] Amelinckx S, Bontinck W, Dekeyser W, Seitz W. Philos Mag 1957;2(15):355-78.

[45] Segall R, Shoaib K. Philos Mag 1970;21(172):713-22.

[46] Dunand D, Mortensen A. Scripta Metall Mater 1991;25(4):761.

[47] Xin X, Daehn G, Wagoner R. Acta Mater 1997;45(5):1821-36.

[48] Cahn J. Nucleation on dislocations. Acta Metall 1957;5:169-72.

[49] Leonard F, Haataja M. Appl Phys Lett 2005;86:181909.

[50] Hin C, Bréchet Y, Maugis P, Soisson F. Philos Mag 2008;88(10):1555-67.

[51] Simon T, Kröger A, Somsen C, Dlouhy A, Eggeler G. Acta Mater 2010;58(5):1850-60.

[52] Hirth J, Pond R. Prog Mater Sci 2011;56(6):586-636.

[53] Zhao Z, Legris A, Thuinet L, Kihn Y, Ambard A, Legras L. J ASTM Int 2008;5(3):1-20.

[54] Thuinet L, Legris A. Acta Mater 2010;58(6):2250-61.

[55] Thuinet L, De Backer A, Legris A. Acta Mater 2012;60(13-14): 5311-21.

[56] Geslin PA, Appolaire B, Finel A. Appl Phys Lett 2014;104(1):011903.

[57] Ahn D, Sofronis P, Minich R. J Mech Phys Solids 2006;54(4):735-55.

[58] Traiviratana S, Bringa E, Benson D, Meyers M. Acta Mater 2008;56(15):3874-86. 